\title{
Os Anais da Academia Brasileira de Ciências e a pesquisa científica no Brasil: estudo exploratório com base no índice-h
}

\author{
Heloísa Maria Ottoni \\ Doutoranda; Centro Brasileiro de Pesquisas Físicas (CBPF), Rio de Janeiro, RJ, Brasil; \\ hottoni@cbpf.br \\ Maria Aparecida Teixeira \\ Mestranda; Universidade Federal do Rio de Janeiro (UFRJ), Rio de Janeiro, RJ, Brasil; \\ aparecida@ccje.ufrj.br \\ Cristiana Amarante \\ Mestre; Instituto de Pesquisas Jardim Botânico do Rio de Janeiro (JBRJ), Rio de Janeiro, RJ, Brasil; \\ cristianaamarante@gmail.com \\ Rosane Castilho \\ Mestre; Pontifícia Universidade Católica do Rio de Janeiro (PUC-Rio), Rio de Janeiro, RJ, Brasil; \\ rosane@inf.puc-rio.br \\ Neusa Cardim \\ Mestre; Universidade do Estado do Rio de Janeiro (UERJ), Rio de Janeiro, RJ, Brasil; \\ ncardims@gmail.com
}

Resumo: Estudo exploratório realizado no periódico Anais da Academia Brasileira de Ciências (AABC) no período de 1966 a 2012. O objetivo foi analisar as variáveis autoria, gênero, afiliação e áreas do conhecimento a que se vinculam os autores de artigos selecionados, identificados na base de dados Web of Science (WoS), através dos valores do índice-h dos artigos deste periódico no período. Os resultados indicaram que os 33 artigos mais citados receberam um total de 1.684 citações, o que representa $18 \%$ de todas as citações recebidas pelos 4.040 artigos identificados pela WoS nos AABC. Neste conjunto de artigos a autoria única é predominante, a maior parte dos autores é do gênero masculino e está afiliada a instituições brasileiras, principalmente universidades; a maior incidência de citações vinculadas à área das Ciências Biomédicas e Médicas. Apesar dos resultados não evidenciarem novidade relevante que altere o perfil da atual produção científica brasileira, buscou-se promover maiores oportunidades de reflexão e discussão dos resultados da pesquisa, analisando peculiaridades relativas a alguns artigos mais citados e estabelecer uma comparação de alguns dados gerais dos AABC com o periódico Proceedings of the National Academy of Sciences of the United States.

Palavras-chave: Análise de citações. Índice-h. Autoria. Produção científica. Periódicos científicos. 


\section{Introdução}

A literatura sobre a história da ciência no Brasil destaca o estabelecimento das universidades, no século XX, como o marco para a consolidação das comunidades científicas. No entanto, já havia no Brasil algumas instituições de pesquisa cuja criação datava de fins do século XIX, como o Instituto Agronômico de Campinas (1887) e o Instituto Butantã (1899), entre outras. Havia, desde então, grupos de cientistas que trocavam conhecimentos e experiências, de forma sistemática (SCHWARTZMAN, 2001). Neste período, algumas revistas brasileiras, como os Arquivos do Museu Nacional, publicada desde 1876, eram lidas no exterior, sendo consideradas como um importante veículo de interação do Brasil com os "centros de produção do saber europeus". (VERGARA, 2004, p. 28).

No início do século XX, as comunidades científicas se expandiram com a criação de sociedades, academias e institutos de pesquisa. Em 1916 surge a Sociedade Brasileira de Ciências (SBC), que em 1921 passa a denominar-se Academia Brasileira de Ciências (ABC), cujos principais objetivos eram dar continuidade ao trabalho científico de seus membros e promover o progresso e/ou difusão da pesquisa e do desenvolvimento tecnológico no país.

As ações da Academia contribuíram efetivamente no processo de desenvolvimento da ciência no País, como a que culminou na criação do Conselho Nacional de Pesquisa (CNPq), em 1951, atual Conselho Nacional de Desenvolvimento Científico e Tecnológico. Muitos cientistas que faziam parte do Conselho Deliberativo do CNPq eram acadêmicos; assim, a participação da Academia nas decisões da política nacional de ciência foi significativa. "Várias instituições importantes, como a Comissão Nacional de Energia Nuclear (CNEN), o Instituto Nacional de Pesquisas Espaciais (INPE) e o Instituto Nacional de Pesquisas da Amazônia (INPA) tiveram sua origem em comitês definidos por esse colegiado". (ACADEMIA BRASILEIRA DE CIÊNCIAS, 2009). 
A primeira administração da SBC definiu como prioridade a publicação de um periódico científico. Em 1917 foi lançada a Revista da Sociedade Brasileira de Ciências, com volumes anuais. Contudo, foi interrompida em dois períodos distintos e retomada duas vezes, com os nomes de Revista de Ciências, em 1920-1921, e Revista da Academia Brasileira de Ciências. Em 1929, a publicação foi regularizada e rebatizada com o nome de Anais da Academia Brasileira de Ciências (AABC), em circulação até os dias de hoje, com periodicidade trimestral (ACADEMIA BRASILEIRA DE CIÊNCIAS, 2009).

Os periódicos têm o papel de repositórios de ideias e, principalmente, instrumentos de preservação da confiabilidade e da autenticação do conhecimento científico. A seleção dos Anais da Academia Brasileira de Ciências $(\mathrm{AABC})$ como objeto de pesquisa privilegiou critérios de tradição e representatividade. Ela é a revista científica brasileira com visibilidade internacional mais antiga em circulação no país com visibilidade internacional e publica a produção de membros da $\mathrm{ABC}$.

Destaca-se sua natureza multidisciplinar, "[...] publicando resultados originais de pesquisas realizadas nas mais diversas áreas da ciência, como matemática, física, química, biológica, biomédica, saúde, sociais, engenharia, agrárias e da terra", produzidos por pesquisadores brasileiros e estrangeiros (ACADEMIA BRASILEIRA DE CIÊNCIAS, 2009, editorial).

A estrutura dos AABC segue a da Academia, constituída por seções que correspondem às áreas do conhecimento, e a partir de 1983, os artigos foram agrupados em grandes áreas de conhecimento, como as citadas acima. A revista, desde a sua criação, possuía uma rede de colaboração internacional (principalmente da França, Alemanha, Inglaterra, República Checa, República Argentina e Chile); a princípio os artigos eram publicados no idioma de origem do autor. Na década de 1990, passou a haver uma recomendação explícita nos fascículos para a adoção do inglês como idioma da publicação; atualmente os textos são publicados, obrigatoriamente, em inglês.

Os AABC hoje em dia estão indexados em bases de dados internacionais, destacando: Current Contents ${ }^{\circledR} /$ Physical, Chemical and Earth Sciences, ISI Alerting Services, Science Citation Index Expanded, BIOSIS, 
Chemical Abstracts, Index Medicus Medline, LILACS, Bibliography and Index of Geology, Institut de L'Information Scientifique et Technique (INIST) e PERIODICA. A seção de Ciências Matemáticas é coberta por Mathematical Reviews e Zentralblatt MATH. Na base Web of Science (WoS), o periódico disponibiliza, no período de 1966 a 1987, apenas os resumos dos artigos e, a partir do ano 2000, o texto integral. A partir do ano 2000, a AABC passa a ser indexada também na base de dados brasileira Scielo (www.scielo.br/aabc).

Este estudo objetiva identificar na base WoS os artigos com maior número de citações recebidas, tendo como ponto de corte o índice h, e neste conjunto, analisar as variáveis autoria (coautoria, gênero e afiliação) e áreas do conhecimento a que se vinculam estes autores, visando identificar informações sobre o perfil de desenvolvimento da pesquisa nos AABC.

\section{Avaliação de periódicos científicos e o índice-h}

$\mathrm{Na}$ avaliação de periódicos científicos, muitos são os indicadores que podem ser aplicados para diferentes propósitos; o resultado de cada estudo pode apresentar diferenças decorrentes da forma de uso dos indicadores (ROUSSEAU, 2002). Indicadores bibliométricos têm sido úteis para avaliar a produção científica.

Dentre os indicadores bibliométricos mais usados atualmente, destacamse o Fator de Impacto e o índice-h, ambos são medidas derivadas da análise de citações. A citação é a relação entre partes do documento citante e partes do documento citado e deve conter primordialmente as informações relativas à autoria, título, local de publicação e data.

O Fator de Impacto, criado por Garfield e Ster, em 1963, é calculado pelo número de citações nos últimos dois anos a um determinado periódico dividido pelo número de artigos publicados no mesmo período de tempo. Como exemplo, tem-se que o Fator de Impacto dos AABC em 2012 foi 0.851 porque o somatório das citações recebidas em 2012, para os artigos publicados nos anos de 2011 e 2012 foi 177 e o total de artigos publicados em 2011 e 2010 foi 208. O fator de impacto é o resultado da divisão 177 por 208, isto é, 0.851 . O fator de impacto é calculado e divulgado anualmente pelo Journal of Citation Report, 
que inclui os principais periódicos mundiais indexados na WoS (GARFIELD, 2006).

O índice-h, criado por Hirsch ${ }^{1}$ em 2005, melhor detalhado a seguir, “[...] é definido como o maior número ' $h$ ' de artigos científicos desse pesquisador que tem pelo menos o mesmo número 'h' de citações” (MARQUES, 2013, p.35). Este indicador é gerado nas bases de dados internacionais WoS e Scopus.

Em dois artigos distintos, produzidos por pesquisadores brasileiros, o índice-h foi aplicado para avaliação do desempenho de cientistas, tendo inclusive os membros da Academia Brasileira de Ciências (ABC) como foco de estudo. Kellner e Ponciano (2008) calcularam os valores do índice-h dos membros titulares da $\mathrm{ABC}$ e cotejaram a produção de cada um com os respectivos currículos apresentados na Plataforma Lattes, encontrando grande variação do índice-h entre os membros da ABC. Na mesma linha de pesquisa, com base neste índice, Mugnaini, Packer e Meneghini (2008) compararam o índice-h dos membros da $\mathrm{ABC}$ e dos membros da National Academy of Sciences of the USA (NAS-USA) para verificar o desempenho relativo de ambos, e observaram, entre outras descobertas, uma variação no índice-h, bem maior nos membros da ABC do que na NAS.

\subsection{O índice-h}

O índice-h surgiu como uma ferramenta para mensurar a qualidade científica de pesquisadores. A sua aplicabilidade no meio científico foi aceita para medir a produtividade e seu possível impacto na carreira científica de um pesquisador; mensura, ao mesmo tempo, a produtividade e o impacto desta produção com base em seus artigos mais citados, porém sem estabelecer um limite temporal (como faz o Fator de Impacto), a contar a partir de sua publicação. Este índice tem sido utilizado com frequência na Cientometria. (MARQUES, 2013, p. 3536)

Ainda segundo Marques (2013), o índice-h de um pesquisador pode ser definido como: 
[...] o maior número " $\mathrm{H}$ " de artigos científicos desse pesquisador que tem pelo menos o mesmo número "H" de citações. Um pesquisador com índice "H" 30 é aquele que publicou pelo menos 30 artigos científicos que foram citados em pelo menos 30 outros trabalhos. A ponderação exclui trabalhos poucos citados. (MARQUES, 2013, p. 35)

Hipoteticamente destaca-se como exemplo um pesquisador que publica 100 artigos indexados na WoS e que tenha recebido 808 citações no total, o seu índice-h é 15 , pois somente 15 de seus artigos receberam, no mínimo, 15 citações.

Evidenciando essa questão numérica díspar com relação a este índice, destaca-se que o índice-h incorpora ao mesmo tempo em seu cálculo a relação entre o fator quantidade (número de artigos publicados por um pesquisador) e o fator qualidade (a demanda de utilização dos artigos publicados por este mesmo pesquisador). Vale ressaltar que este índice, por si só, não determina o reconhecimento de um pesquisador num universo de pesquisa, mas é um instrumento a mais para análise, avaliação e tomada de decisão sobre pontos de vista vinculados à política e ao desempenho da produção científica. Considerando um pesquisador que publique apenas um artigo que é citado infinitas vezes, o artigo tem valor científico, é meritoso, apesar de seu índice-h ser 1.

O que se ressalta é que o uso de indicadores depende da finalidade a que se propõe alcançar. Segundo Rousseau (2002) há vários indicadores científicos que podem ser aplicados a diferentes propósitos; o resultado de cada estudo pode apresentar diferenças decorrentes da forma de uso dos indicadores.

Reiterando este tema, Costas e Bordons (2007, p. 431) mencionam que a principal vantagem do índice-h "[...] é que combina em um único indicador uma medida de quantidade e outra de impacto de produção". O termo "medida de quantidade" se refere ao número de trabalhos publicados e o termo "impactos de produção" faz referência ao número de trabalhos citados.

A repercussão do índice-h se evidencia na cultura de publicação de várias áreas do conhecimento no Brasil na atualidade. Tanto é assim que ele é 
usado pela Coordenação de Aperfeiçoamento de Pessoal de Nível Superior (Capes) como um dos critérios para definir o estrato mais alto de revistas científicas em diversas áreas, no sistema Qualis. Segundo informa Egghe (2010), o índice-h tem sido também aplicado no contexto de livros, patentes, instituições e países.

Por outro lado, segundo argumenta Marques (2013), autores como Rogério Meneghini, Rogério Mugnaini e até o próprio idealizador do índice-h, Jorge Hirsch, destacam limitações neste indicador. O fato é que, apesar de suas vantagens, o índice-h como único indicador causa prejuízo ao pesquisador que prima pela qualidade ao invés da quantidade, isto é, publica pouco, mas sua produção é muito citada e mesmo assim o seu índice-h é baixo.

Outro fator que interfere na avaliação dos resultados do índice-h é que nem sempre os citados e os citantes publicam em periódicos de impacto em suas áreas de conhecimento. Seria conveniente que se agregasse um componente qualitativo de valorização ao periódico ao índice-h, já que este índice privilegia o fator qualidade em seu cálculo.

Alerta-se também que podem ocorrer discrepâncias no resultado do índice-h se os artigos citados não estiverem contextualizados em sua área de conhecimento, já que áreas distintas tendem a possuir perfis díspares de resultados numa análise, os quais certamente irão interferir neste indicador, principalmente na atualidade, onde o padrão de produção e de visibilidade científica varia de área para área, em função também da inter e transdisciplinaridade.

Também é equivocado comparar o desempenho de pesquisadores de áreas diferentes e em estágios diferentes da carreira, pois evidentemente, apesar de suas competências serem igualmente reconhecidas, seus índices-h serão diferentes por determinação do tempo percorrido de produção científica de um pesquisador e do perfil variado de desempenho de cada área científica.

E acrescenta-se ainda que, no caso de grupos de pesquisa, esse indicador acaba sendo impreciso porque não há como quantificar a incidência de participação nos artigos por cada um de seus autores. 
Mas mesmo considerando essas limitações (e possíveis outras não identificadas neste momento), o índice-h aplicado às revistas científicas tem se revelado forte concorrente ao Fator de Impacto (EGGHE, 2010, p. 78). Ao contrário deste - baseado no número total de citações ao periódico durante um determinado ano -, o índice-h é calculado com base no número de citações que os artigos recebem individualmente desde a data de sua publicação, sem limitação de tempo, evidenciando não só a produção, mas também sua demanda entre pares.

Entende-se, portanto, que o índice-h é mais um indicador de produtividade científica disponível dentre outros; reconhece-se que ele não é determinante se analisado isoladamente. Nem sempre o índice-h oferece, por si só, um resultado final de desempenho de um pesquisador, mas possui alto valor numa avaliação quando combinado com outros parâmetros que atendam a cada objetivo específico perseguido, considerando-se o contexto de suas limitações.

No presente trabalho, que visa levantar informações sobre o cenário da pesquisa brasileira, foi utilizado o índice-h dos AABC, gerado pela base WoS, que é 33. Esse periódico tem 33 artigos que foram citados no período analisado, cada um deles pelo menos 33 vezes.

\section{Procedimentos metodológicos}

A pesquisa realizada é exploratória, visto que seu objetivo é identificar e caracterizar o objeto de estudo; tem a finalidade de esclarecer "[...] conceitos e idéias, tendo em vista a formulação de [...] hipóteses pesquisáveis para estudos posteriores" em função do relacionamento entre as variáveis identificadas (GIL, 2008, p. 27). Foram utilizadas nesta pesquisa as ferramentas bibliométricas de análise de citações e também o índice-h.

A fonte de pesquisa utilizada foi o periódico Anais da Academia Brasileira de Ciências (AABC), sendo o universo da pesquisa restrito a artigos desses anais entre os anos de 1966 a 2012. Sua unidade de análise foi o conjunto de artigos mais citados na base de dados WoS da Thomson Reuters. Considerou- 
se o número de artigos indexados e o número de citações recebidas, tendo o índice-h como delimitador nesta seleção.

A unidade de análise se constituiu em um conjunto de 33 artigos dos $\mathrm{AABC}$, que foram analisados segundo as variáveis: áreas do conhecimento, dados de autoria (incluindo gênero e afiliação) e natureza e nacionalidade das instituições.

O desenvolvimento da pesquisa nos $\mathrm{AABC}$ contemplou cinco etapas, na ordem a seguir: 1) recuperação de todos os artigos da $\mathrm{AABC}$ indexados na base WoS entre os anos de 1966 e 2012; 2) seleção da unidade de análise dos artigos mais citados deste conjunto recuperado, com base no índice-h gerado pela base WoS; 3) listagem das referências dos artigos selecionados em ordem decrescente de número de citações; 4) análise desta listagem com foco nas autorias e nas áreas do conhecimento dos artigos selecionados e 5) avaliação dos resultados, estabelecendo correlações entre estas variáveis, comparação com fonte similar dos USA e comentários críticos.

\section{Os AABC na WoS e o índice-h: resultados e discussão}

Entre os anos de 1966 a 2012 (45 anos de pesquisa) foram indexados na base WoS 4.040 artigos dos AABC que receberam 9.472 citações, uma média de 2,3 citações por artigo; média considerada baixa se comparada ao padrão médio de 12 citações de artigo por artigo científico, estimado por Price em seu clássico artigo Network of scientific papers, de 1965. Ampliando informações sobre o desempenho da produção científica, destaca-se que o periódico Proceedings of the National Academy of Sciences of the United States (PNAS), com cobertura de análise no período de 1945-1951 e 1961-2013 (58 anos de pesquisa) possui um índice-h de 529; e entre 1999 a 2013 o periódico publicou um total de 110 mil artigos com uma média acima de 10 citações por artigo. (SCIMAGO JOURNAL RANK, 2014).

Vale uma reflexão sobre vários fatores que justificam as diferenças do índice-h entre os periódicos das academias de ciências americana (529) e brasileira (33). A periodicidade dos AABC é trimestral e o PNAS semanal e, 
portanto, este tende a publicar mais artigos que os $\mathrm{AABC}$, variável que se reflete no cálculo do índice-h; vale também refletir sobre o fato de os $\mathrm{AABC}$, principalmente nas décadas de 1960 e 1970, publicarem quase que exclusivamente artigos contendo somente os resultados de pesquisas científicas dos membros da Academia Brasileira de Ciências, sendo que muitos desses artigos apresentavam pouca incidência de citações, em alguns casos nenhuma, o que tende a "puxar" a média de citações dos artigos do periódico para baixo.

Sob uma análise geral entre os dois periódicos, a ampla diferença neste índice também se justifica pela reconhecida diferença de perfil de desenvolvimento econômico entre o Brasil e os Estados Unidos, que se reflete na produção científica.

Foram escritos em língua inglesa 60,8\% (2.455) dos artigos estudados publicados pela AABC, e 33\% (1.349) na língua portuguesa. Analisando-se o histórico dos $\mathrm{AABC}$, considerando que a obrigatoriedade de escrita dos artigos em inglês aconteceu somente a partir de 1990, pode-se perceber que em 24 anos de publicação (1966-1990) o periódico produziu 33\% dos artigos escritos em português, enquanto que, comparativamente entre 1990 e 2012, em 22 anos de produção, gerou aproximadamente $90 \%$ de artigos em inglês, o que demonstra que houve um aumento relevante na produção de artigos no periódico num período menor de tempo, nos anos mais recentes analisados; os restantes, 6,2\% do total, foram escritos em espanhol, francês, italiano e alemão, línguas de membros estrangeiros e correspondentes internacionais associados à $\mathrm{ABC}$.

A maior parte dos autores (73\%) é filiada a instituições brasileiras, e os demais (27\%) a um conjunto de instituições de origem americana, francesa, alemã, argentina, canadense, tcheca, inglesa, chilena, italiana, espanhola e japonesa, o que, de certa forma, demonstra haver um esforço na internacionalização do periódico e na possível ampliação da busca por participação de estrangeiros nas iniciativas da $\mathrm{ABC}$ ao longo do tempo.

\subsection{Análises das citações}


Os 33 artigos selecionados e que fazem parte da unidade de análise correspondem a $0,82 \%$ do total de artigos indexados. Estes artigos receberam um total de 1.684 citações, o que representa $18 \%$ de todas as citações recebidas pelos 4.040 artigos dos AABC no período de 1996 a 2012, e se concentraram entre os anos de 1966 a 2007.

O Anexo A detalha em tabela os resultados globais evidenciados pela análise das citações feita ao conjunto destes 33 artigos dos $\mathrm{AABC}$, sendo identificado na tabela o número de citações recebidas por cada um dos artigos, acrescido das variáveis ano de publicação, número de autores, países de origem da produção e áreas do conhecimento, detalhadas adiante na metodologia.

Nota-se uma concentração de artigos citados após o ano 2000 (64\%) e também se destaca que não foram encontrados artigos na unidade de análise entre os anos de 2008-2012 que estivessem também entre os mais citados do periódico.

Com relação a essa queda de produção entre 2008 e 2012, é provável que a crise econômica mundial deflagrada entre os anos 2008 e 2009, principalmente nos Estados Unidos, tenha interferido no desempenho da ciência brasileira no período, deixando sequelas inclusive nos anos seguintes. Reiterando a questão, destaca o ex-reitor da Unicamp e diretor científico da Fundação de Amparo à Pesquisa do Estado de São Paulo (FAPESP), Carlos Henrique de Brito Cruz (2013) em entrevista ao Jornal da Ciência, que "O número de artigos científicos com autores do Brasil vem crescendo, embora a uma taxa menor nos últimos três anos". Em momentos da entrevista, Cruz se pronuncia informando que os cortes de orçamento de 2011/2012 no país também afetaram o sistema, especialmente o acadêmico. Destaca que:

Em 2010 e 2011 teve cortes importantes no orçamento do MCTI [Ministério da Ciência, Tecnologia e Inovação]. Houve um importante decréscimo na capacidade cientifica e tecnológica no Brasil, por causa da crise de 2009 e dos erros na política econômica, como câmbio, que resultaram na assim chamada desindustrialização. Quando a indústria fica mais fraca, diminui a capacidade de ter pesquisa na indústria, o que se reflete de imediato na baixa produtividade e baixa competitividade. (CRUZ, 2013, documento eletrônico não paginado). 
E mais um dado peculiar nesse conjunto dos 33 artigos é que os quatro artigos mais citados no ano de 1984 encontram-se em um único fascículo. O fascículo é temático, trata do fenômeno da depressão alastrante que afeta o sistema nervoso central, descoberto pelo biólogo e pesquisador Aristides Azevedo Pacheco Leão, fenômeno que recebeu o seu nome e se tornou conhecido internacionalmente como Leão's spreading. Cientistas foram convidados a escrever sobre o assunto em evento comemorativo em homenagem a Aristides Leão, que foi um dos acadêmicos da $A B C$ e que presidiu esta Academia por 15 anos. Pesquisadores do Brasil, da Dinamarca, da Tchecoslováquia e dos Estados Unidos da América foram os produtores desses artigos. Esta evidência comprova o esforço da $\mathrm{ABC}$ em expandir o contato e o intercâmbio do conhecimento entre cientistas como estratégia para ampliação da produção científica. É provável que eventos comemorativos no âmbito da ciência, em todos os tempos, continuem a ser motivo de pronunciamento de pesquisadores, principalmente numa situação como essa, em homenagem a um ex-presidente de associação científica, de renome internacional. Vale lembrar que na década de 1980 o fenômeno da globalização ainda não estava institucionalizado e a tecnologia da informação ainda não oferecia as facilidades de acesso eletrônico e comunicação virtual à troca de ideias e informação entre os cientistas. As dificuldades de comunicação da ciência tendiam a ser maiores e divergiam das existentes hoje em dia. Na década de 1980 no Brasil e no mundo ainda predominava o acesso e a repercussão do trabalho científica dos pesquisadores através de cartas, de visitas pessoais e da afiliação a academias científicas, que colaboravam para mobilizar e integrar os pesquisadores e seus grupos de pesquisa.

Os cinco artigos mais citados receberam acima de 68 citações: três deles datam do século XXI, e os outros dois do século passado. Ressalta-se que quatro destes artigos foram de autoria única, e um possui cinco autores. Em relação às afiliações dos seis pesquisadores destacados, cinco têm origem no Brasil e quatro nos EUA, sendo que um dos artigos resulta de parceria Brasil e EUA. Destes cinco artigos, três pertencem à área das Ciências Biomédicas e Médicas, os outros dois às áreas Ciências Biológicas e Ciências Matemáticas. (Quadro 6). 
Quando um artigo recebe mais de 100 citações na WoS, é classificado como um artigo highly cited. Com base neste critério, dois artigos se enquadram nesta classificação (Quadro 1). O mais citado deles foi escrito por um pesquisador brasileiro, médico, bolsista de produtividade em pesquisa do $\mathrm{CNPq}$ nível 1A, com pos-doc pela Universidade Paris Decartes em Anatomia Humana e é afiliado à Universidade do Estado do Rio de Janeiro (UERJ). O artigo tem como temática a instrumentação médica e apresenta uma revisão das principais ferramentas de estereologia, útil a obtenção de uma base de informação para cientistas não-especializados sobre amostra de cortes em Patologia e Neurociências.

O outro artigo é de autoria coletiva de brasileiros da Embrapa da área de Agrobiologia, alguns com bolsa de produtividade científica do $\mathrm{CNPq}$, com um norte-americano e uma tcheco. Sua temática é agrobiologia voltada à fixação de nitrogênio no solo através de pesquisa com a bactéria Azospirillum, visando incrementar a produtividade do agronegócio. Deste grupo de autores, destaca-se Johanna Liesbeth Kubelka Döbereiner, tcheca de nacionalidade e naturalizada brasileira em 1956, engenheira agrônoma, que atuou na Embrapa (Seropédia, RJ) até o ano 2000, quando faleceu; ela foi considerada “[...] a sétima cientista brasileira mais citada pela comunidade científica mundial, segundo levantamento de 1995 divulgado na Folha de S. Paulo" (GIFALLI, 2014, documento eletrônico não paginado). Destaca-se, como reflexão, o valor dessa contribuição científica ao país, de reconhecimento internacional para o gênero feminino no século $\mathrm{XX}$, quando ainda predominava o preconceito quanto à atuação da mulher na ciência mundial, inclusive no Brasil. Certamente Döbereiner deixou o seu legado de conhecimento no país e participou da formação científica de muitos brasileiros, além de se distinguir na participação de um artigo highly cited produzido no Brasil.

O objetivo científico envolvendo a temática destes dois artigos highly cited convergem quanto à eficiência de procedimentos de uso técnico científicos e a preocupação com a otimização de custos e benefício social em sua adoção. 

pesquisa científica no Brasil: estudo exploratório com base no índice-h

Heloísa Maria Ottoni, Maria Aparecida Teixeira, Cristiana Amarante, Rosane Castilho, Neusa Cardim

Quadro 1 - Análise de citações dos AABC (1966-2012) - artigos highly cited

\begin{tabular}{|c|l|l|}
\hline $\begin{array}{c}\text { Cit. } \\
\text { Receb. }\end{array}$ & \multicolumn{1}{|c|}{ Títulos dos artigos } & \multicolumn{1}{c|}{ Autores } \\
\hline $\begin{array}{c}132 \\
(2003)\end{array}$ & $\begin{array}{l}\text { Stereological tools in biomedical } \\
\text { research }\end{array}$ & $\begin{array}{l}\text { C. A. Mandarim-de-Lacerda } \\
117\end{array}$ \\
$\begin{array}{l}\text { A new acid-tolerant Azospirillum } \\
\text { species }\end{array}$ & $\begin{array}{l}\text { F. M. Magalhães; J. .I. } \\
\text { Baldani; S. M. Souto; J. R. } \\
\text { Kuykendall; J. Dobereiner }\end{array}$ \\
\hline
\end{tabular}

Fonte: dados da pesquisa.

\subsection{Autoria}

Pelo índice-h, o estudo totalizou 88 registros de autoria, sendo de 86 autores diferentes; 13 artigos (40\%) de autoria única, sendo, desse conjunto, 54\% da área de Ciências Biomédicas e Médicas. A autoria dupla foi identificada em oito artigos (24\%), e os 12 artigos restantes (36\%) desse total foram escritos por três autores ou mais.

Figura 1 - Análise de citações nos AABC (1966-2012) - modalidades de autoria

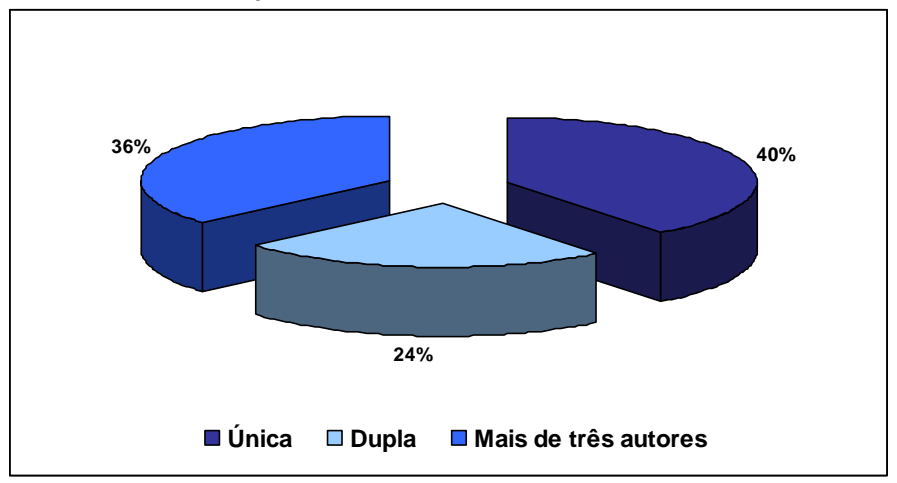

Fonte: dados da pesquisa.

$\mathrm{O}$ artigo com o maior número de autores, oito, da área de ciências da terra, resulta de parceria entre pesquisadores do Brasil e da Itália. Dois pesquisadores têm mais de um artigo selecionado, ambos com dois artigos na área de ciências da terra: Alexander Wilhelm Armin Kellner, que é membro da ABC, geólogo nascido no Principado de Liechtenstein, doutor pelo Departamento de Ciências Geológicas do Laboratório Geológico LamontDoherty da Universidade de Colúmbia e Museu Americano de História Natural e José Ivo Baldani, brasileiro, engenheiro agrônomo, pesquisador da Embrapa com Doutorado em Ciências do Solo pela Universidade do Texas (A\&M 
University); inclusive Baldani também é um dos autores de artigo high cited. Como reflexão, destaca-se que o doutorado no exterior ainda é um quesito que no Brasil tende a promover o trabalho de um pesquisador.

Apesar da área de ciências da Terra não estar no topo das áreas mais citadas nesta pesquisa, ela assume relevância pela titulação destes dois autores e por suas publicações estarem entre os artigos mais citados dos AABC.

A maior parte dos autores é do gênero masculino (68\%, e $31 \%$ do gênero feminino). Resultado que pode ser interpretado como um reflexo da menor participação feminina nas Ciências (HAYASHI, 2013).

A predominância do gênero masculino nas autorias reflete a situação do gênero no país. Em estudo de Leta e colaboradores (2006) ficou constatado que:

Muito embora as estatísticas apontem nitidamente uma mudança no gênero dos pesquisadores brasileiros, percebe-se ainda que as pesquisadoras no Brasil ainda são minoria entre: 1) os pesquisadores premiados com a bolsa de produtividade do $\mathrm{CNPq}$; 2) os pesquisadores-líderes de grupos de pesquisa; 3) os pesquisadores nomeados para os comitês assessores das agências de fomento; 4) os docentes nomeados para cargos de chefias; e 5) os nomeados para a Academia Brasileira de Ciências. (LETA et al., 2006, p. 538).

A pesquisa deste estudo para identificação do gênero foi realizada em bases de dados científicas diversas, uma vez que a maioria dos nomes dos autores apresenta o sobrenome e as iniciais do prenome; um dos autores não teve o seu gênero identificado.

Foi mapeada a afiliação dos pesquisadores a institutos, universidades e laboratórios de pesquisa, segundo informa o Quadro 2. Do conjunto dos 86 autores, apenas um não possuía dados de afiliação, possivelmente por não ter Currículo Lattes, sendo de origem estrangeira. Dos pesquisadores, $51(59,3 \%)$ possuem afiliação a instituições brasileiras, e os demais 35 (40,7\%) a instituições estrangeiras. Os pesquisadores brasileiros estão afiliados a 16 instituições e estão majoritariamente concentrados em universidades (11 das 16 instituições), lideradas em número de afiliados deste estudo pela USP e UFRJ, seguidas pela FIOCRUZ. A organização internacional QS Quacquarelli 

pesquisa científica no Brasil: estudo exploratório com

Symonds University Rankings colocou a USP em $1^{\circ}$ lugar no ranking das universidades no Brasil e em $2^{\circ}$ na América Latina.

Percebe-se nesta pesquisa que, do grupo de instituições brasileiras relacionadas à afiliação dos autores, apenas quatro são centros de pesquisa(Quadro 2). Destaca-se que as universidades continuam a cumprir o papel prioritário da Pesquisa e Desenvolvimento (P\&D) no país, em detrimento dos centros de pesquisa e empresas, que deveriam ocupar este espaço, como acontece na maioria dos países desenvolvidos do mundo.

Quadro 2 - Análise de citações nos AABC (1966-2012) - instituições brasileiras relacionadas à afiliação de pesquisadores

\begin{tabular}{|l|c|}
\hline \multicolumn{1}{|c|}{ Instituições brasileiras } & $\begin{array}{c}\mathbf{N}^{\text {o. }} \text { de pesquisadores } \\
\text { afiliados }\end{array}$ \\
\hline Universidade de São Paulo (USP) & 18 \\
\hline Universidade Federal do Rio de Janeiro (UFRJ) & 10 \\
\hline Fundação Oswaldo Cruz (FIOCRUZ) & 4 \\
\hline Universidade do Estado do Rio de Janeiro (UERJ) & 3 \\
\hline Empresa Brasileira de Pesquisa Agropecuária (EMBRAPA) & 3 \\
\hline Universidade Federal do Ceará (UFC) & 2 \\
\hline Universidade de Brasília (UnB) & 2 \\
\hline Universidade Federal Fluminense (UFF) & 1 \\
\hline Universidade Federal do Pará (UFPa) & 1 \\
\hline Universidade Federal de São Paulo (UniFESP) & 1 \\
\hline Universidade Federal de Lavras & 1 \\
\hline Universidade de Campinas (UNICAMP) & 1 \\
\hline Instituto Nacional do Câncer (INCA) & 1 \\
\hline Escola Bahiana de Medicina e Saúde Pública & 1 \\
\hline Departamento Nacional Pesquisa Mineral (DNPM) & 1 \\
\hline Universidade Federal do Rio Grande do Sul (UFRGS) & 1 \\
\hline TOTAL & 51 \\
\hline
\end{tabular}

Fonte: dados da pesquisa.

Com relação à afiliação dos pesquisadores estrangeiros deste conjunto de artigos, três instituições destacaram-se no quantitativo de afiliados: a University of Texas Medical Branch, a University of Trieste e a Czechoslocak Academy of Sciences, com primazia das universidades, como foi apontado na análise da afiliação brasileira. 

pesquisa científica no Brasil: estudo exploratório com

Considerando-se o país de origem dessa produção (Quadro 3), como esperado, o Brasil lidera o grupo e aparece como o único país de origem na produção de 17 artigos (51,6\%). No entanto, a parceria e a presença de outros países é um indicador relevante (Quadro 4), o que acontece com predominância da área de ciências da terra e tende a se constituir em grupos de pesquisa envolvendo pesquisadores visitantes de instituições públicas e pesquisadores estrangeiros, principalmente quando membros da $\mathrm{ABC}$.

Quadro 3 - Análise de citações nos AABC (1966-2012) - países de origem e artigos produzidos

\begin{tabular}{|l|c|}
\hline \multicolumn{1}{|c|}{ País } & Artigos \\
\hline Brasil & 17 \\
\hline EUA & 6 \\
\hline Brasil e EUA & 2 \\
\hline Reino Unido & 2 \\
\hline Brasil e Argentina & 1 \\
\hline Brasil e Austrália & 1 \\
\hline Brasil e Itália & 1 \\
\hline Chile & 1 \\
\hline Dinamarca & 1 \\
\hline Tchecoslováquia & 1 \\
\hline \multicolumn{2}{|c|}{ Fonte: dados da pesquisa. } \\
\hline
\end{tabular}

Quadro 4 - Análise de citações nos AABC (1966-2012) - artigos produzidos em parceria do Brasil com outros países

\begin{tabular}{|c|c|c|c|l|l|}
\hline Art. & Ano & $\begin{array}{c}\text { N. de } \\
\text { citações }\end{array}$ & Autores & \multicolumn{1}{|c|}{ País } & \multicolumn{1}{|c|}{$\begin{array}{c}\text { Área do } \\
\text { conhecimento }\end{array}$} \\
\hline $\mathbf{2}$ & 1983 & 117 & 5 & Brasil / EUA & Ciências biológicas \\
\hline $\mathbf{2 1}$ & 2007 & 41 & 4 & Brasil/Argentina & Ciências da terra \\
\hline $\mathbf{2 2}$ & 2002 & 39 & 5 & Brasil / Austrália & Ciências da terra \\
\hline $\mathbf{2 6}$ & 1976 & 37 & 2 & Brasil / EUA & Ciências da terra \\
\hline $\mathbf{2 9}$ & 1983 & 36 & 8 & Brasil / Itália & Ciências da terra \\
\hline
\end{tabular}

Fonte: dados da pesquisa.

Do total de 11 artigos com titulação de estrangeiros (Quadro 4) há destaque dos EUA, com a produção de $33 \%$ do conjunto dos artigos analisados. 

pesquisa científica no Brasil: estudo exploratório com

Entre os dez artigos mais citados nesta análise, os EUA se destacam com seis destes artigos (18,2\%) e com os maiores índices de citação em áreas do conhecimento variadas (Quadro 5). No conjunto de artigos internacionais foram identificados também outros países: Reino Unido, Argentina, Austrália, Chile, Dinamarca, Itália e Tchecoslováquia. Destaca-se também, em relação ao Quadro 4, a participação dos Estados Unidos em artigo highly cited, e convém destacar para reflexão que possivelmente estes países deixam uma marca significativa para a rede de produção científica brasileira e a internacionalização e expansão desse conhecimento para fora do país, considerando a possível repercussão dessa produção ao se estabelecer entre os artigos mais citados em momentos variados do século XX e XXI.

Quadro 5 - Análise de citações nos AABC (1966-2012) - artigos publicados por estrangeiros

\begin{tabular}{|c|c|c|c|c|c|}
\hline Art. & Ano & $\begin{array}{c}\text { N.de } \\
\text { citações }\end{array}$ & Autores & País & Área do conhecimento \\
\hline $\mathbf{4}$ & 1970 & 72 & 1 & EUA & Ciências matemáticas \\
\hline $\mathbf{5}$ & 2001 & 68 & 1 & EUA & Ciências biomédicas e médicas \\
\hline $\mathbf{1 2}$ & 2005 & 50 & 1 & Reino Unido & Ciências biomédicas e médicas \\
\hline $\mathbf{1 6}$ & 2004 & 47 & 1 & EUA & Ciências biológicas \\
\hline $\mathbf{1 8}$ & 2005 & 46 & 1 & Reino Unido & Ciências biomédicas e medicas \\
\hline $\mathbf{2 0}$ & 1984 & 44 & 1 & EUA & Ciências biomédicas e médicas \\
\hline $\mathbf{2 4}$ & 1981 & 38 & 5 & Chile & Ciências da terra \\
\hline $\mathbf{2 5}$ & 1984 & 37 & 3 & Tchecoslováquia & Ciências biomédicas e médicas \\
\hline $\mathbf{2 7}$ & 2005 & 36 & 5 & EUA & Ciências biomédicas e médicas \\
\hline $\mathbf{3 2}$ & 2003 & 33 & 2 & EUA & Ciências da terra \\
\hline $\mathbf{3 3}$ & 1984 & 33 & 2 & Dinamarca & Ciências biomédicas e médicas \\
\hline
\end{tabular}

Fonte: dados da pesquisa.

\section{3 Áreas de conhecimento}

Quanto ao número de artigos publicados por área do conhecimento, houve predominância das Ciências Biomédicas e Médicas, com 16 artigos (48,5\%) e 841 citações (49,6\%); e das Ciências da Terra, com 10 artigos (30,3\%) e 393 citações $(23,3 \%)$, de reconhecida tradição de pesquisa no país. A seguir, com cinco artigos (15,2\%) e 330 (19,6\%) citações, identificou-se a área das Ciências Biológicas, seguida pelas demais áreas: Ciências Matemáticas e Ciências 

pesquisa científica no Brasil: estudo exploratório com base no índice-h

Heloísa Maria Ottoni, Maria Aparecida Teixeira, Cristiana Amarante, Rosane Castilho, Neusa Cardim

Agrárias, com um artigo cada (3\% da produção), tendo a primeira 72 (2,9\%) citações e a segunda 48 (4,2\%) citações. (Quadro 6).

Quadro 6 - Análise de citações nos AABC (1966-2012) - áreas do conhecimento, artigos analisados e citações

\begin{tabular}{|c|c|c|c|}
\hline Áreas do conhecimento & $\begin{array}{c}\text { Número de } \\
\text { Artigos }\end{array}$ & $\begin{array}{c}\text { Citações } \\
\text { totais }\end{array}$ & $\begin{array}{c}\text { Média } \\
\text { citações/artigo }\end{array}$ \\
\hline Ciências Biomédicas e Médicas & 16 & 841 & 52 \\
\hline Ciências da Terra & 10 & 393 & 39 \\
\hline Ciências Biológicas & 5 & 330 & 66 \\
\hline Ciências Matemáticas & 1 & 72 & 72 \\
\hline Ciências Agrárias & 1 & 48 & 48 \\
\hline Total & 33 & 1.684 & 277 \\
\hline
\end{tabular}

Fonte: dados da pesquisa.

Neste mesmo quadro 6, considerando-se o cálculo médio de citações pelo número de artigos publicados, por área do conhecimento, tem-se a área de ciências matemáticas e ciências biológicas no topo das áreas com maior demanda de citações, individualmente, por artigo. Porém o foco desta análise é a demanda de artigos.

As áreas que tem mais artigos dentro do conjunto analisado reforçam outros estudos da literatura que afirmam que a área da Saúde apresenta maior quantitativo de pesquisas publicadas. No estudo de Kellner e Ponciano (2008), que avaliou o desempenho dos membros da $\mathrm{ABC}$ em relação ao índice-h, se verificou que as maiores médias desse indicador foram encontradas nas Ciências Biomédicas, da Saúde e Químicas. Mugnaini; Packer e Meneghini (2008) concluíram ser a Biomedicina uma área forte em relação à produção brasileira na literatura científica.

\section{Considerações finais}

Os resultados desta pesquisa nos $\mathrm{AABC}$ não evidenciou novidade relevante que altere o perfil da atual produção científica brasileira. Em função desta constatação, buscou-se neste artigo abrir um espaço para reflexão e discussão ao longo do texto sobre peculiaridades identificadas em alguns artigos do conjunto analisado que gerasse conhecimento sobre o cenário científico brasileiro. Foram 
também feitas algumas comparações de dados gerais dos $\mathrm{AABC}$ com os do Proceedings of the National Academy of Sciences of the United States, que se refletem na diferença nos números levantados. Foram evidenciadas algumas peculiaridades que particularizam os dois periódicos, dentre elas o estágio de desenvolvimento econômico em que se encontram os países Brasil e Estados Unidos.

Um trabalho de pesquisa sobre um cenário nacional da ciência envolvendo ferramentas cientométricas, como neste caso a pesquisa científica no Brasil e o índice-h, acaba gerando uma "aventura investigativa" praticamente infindável.

O olhar sobre os números desta pesquisa, por cinco autores bibliotecários, com afiliação à universidades e centros de pesquisa, expande os caminhos e possibilidades de se gerar conhecimento novo e enriquecer seus resultados. A cientometria foi empiricamente percebida como uma ferramenta adequada à temática da pesquisa, proporcionando eficácia e aumento de acuidade sobre os resultados encontrados neste estudo, motivando a evolução da pesquisa.

O índice-h, especificamente neste estudo que contempla a produção científica também em português, motiva uma contestação à qual não foi possível se responder. O fato da WoS privilegiar artigos escritos em inglês pode, de certa forma, ter interferido para diminuir o valor desse índice, visto que somente a partir de 1990 os AABC passam a ter seus artigos obrigatoriamente escritos em inglês, ainda que o período analisado date de 1966. Uma pesquisa em bases nacionais brasileiras não foi feita neste estudo.

Percebeu-se também que seria oportuna a evolução de pesquisas no Brasil com foco no gênero, aprofundando o estudo sobre o mapeamento da evolução científica de desempenho feminino na ciência brasileira, considerando as variáveis área e subáreas do conhecimento, em análise de citações. Acreditase que seriam gerados resultados interessantes e importantes que poderiam ser contextualizados em âmbito mundial a partir de pesquisa em alguns periódicos de impacto científico, como o próprio PNAS aqui mencionado, entre outros. 

pesquisa científica no Brasil: estudo exploratório com

Fica evidente o processo de internacionalização dos $\mathrm{AABC}$ e da pesquisa científica brasileira. Dos artigos analisados 70\% foram em inglês e $73 \%$ deles tem afiliação nacional, priorizando a USP como a maior produtora dos artigos. É possível que este desempenho se justifique pelo orçamento do Estado de São Paulo destinado ao fomento à pesquisa e à educação, inclusive quanto a parcerias estrangeiras, ser maior que as demais instituições no país.

Foi constatado que a colaboração científica internacional do Brasil não foi muito expressiva pelos AABC. Apenas $15 \%$ do conjunto de artigos analisado teve coautorias internacionais. Pela WoS, o Brasil colaborou com instituições de apenas 5 países estrangeiros (destacando os Estados Unidos e Inglaterra) e apenas uma colaboração "sul-sul", com o Chile. Um dos fatores limitantes pode ser a barreira da língua e a dificuldade financeira e operacional do ambiente de pesquisa no país em promover o intercâmbio internacional entre pesquisadores. Os principais estímulos ao fomento à pesquisa oferecidos pelos fundos setoriais e programas como o CT-Infra e demais iniciativas promovidas pelo Governo brasileiro de apoio a pesquisa envolvendo $\mathrm{RH}$, podem ter se refletido na produção científica dos $\mathrm{AABC}$, e seria interessante que fossem investigados em futuras pesquisas.

A análise das cocitações a este conjunto de artigos selecionados também geraria estudos interessantes, valeria a pena se ampliar as possibilidades de estudo neste sentido.

\section{Referências}

ACADEMIA BRASILEIRA DE CIÊNCIAS. História. 2009. Disponível em: <http://www.abc.org.br/article.php3?id_article=4>. Acesso em: 25 jan. 2015.

COSTAS, R.; BORDONS, M. Una visión crítica del índice h: algunas consideraciones derivadas de su aplicación práctica. El Profesional de la Información, Barcelona, v. 16, n. 5, 2007. Disponível em: <http://www.elprofesionaldelainformacion.com/contenidos/2007/septiembre/04.pdf >. Acesso em: 25 jan. 2015.

CRUZ, C. H. B. É preciso buscar maior impacto da ciência que é feita no Brasil. 2013. Disponível em: 
<http://www.sbpcnet.org.br/site/noticias/materias/detalhe.php?id=1896>.

Acesso em: 18 mar. 2015.

EGGHE, L. The Hirsch Index and related impact measures. Annual Review of Information Science and Technology, White Plains, v.44, p.1-69, 2010.

GARFIELD, E. The history and meaning of the journal impact factor. JAMA: the Journal of the American Medical Association, Chicago, v. 295, n. 1, p. 9093, 2006.

GIFALLI, M. Johanna Döbereiner. 2014. Disponível em:

<http://www.iea.usp.br/pessoas/expositores/johanna-dobereiner>. Acesso em 08 jun. 2015.

GIL, A. C. Métodos e técnicas de pesquisa social. 6. ed. São Paulo: Atlas, 2008.

HAYASHI, C. R. M. Métricas da participação feminina na ciência e tecnologia no contexto dos INCT's: primeiras aproximações. Liinc em Revista, Rio de Janeiro, v. 9, n. 1, p. 143-170, maio 2013.

KELLNER, A. W. A.; PONCIANO, L. C. M. O. H-index in the Brazilian Academy of Sciences: comments and concerns. Anais da Academia Brasileira de Ciências, Rio de Janeiro, v. 80, n. 4, dez. 2008. Disponível em:

<http://dx.doi.org/10.1590/S0001-37652008000400016>. Acesso em: 30 jan. 2015.

LETA, J. et al. As mulheres na pesquisa, no desenvolvimento tecnológico e na inovação: uma comparação Brasil / França. Revista do Serviço Público, Brasília, v. 57, p. 531-547, 2006.

MARQUES, F. Os limites do índice-h: supervalorização do indicador que combina quantidade e qualidade da produção científica gera controvérsia. São Paulo: Revista Pesquisa FAPESP, São Paulo, n. 207, p. 35-39, maio 2013.

MUGNAINI, R., PACKER, A. L.; MENEGHINI, R. Comparison of scientists of the Brazilian Academy of Sciences and of the National Academy of Sciences of the USA on the basis of the $h$-index. Brazilian Journal of Medical and Biological Science, Ribeirão Preto, v. 41, n. 4, p. 258-262, 2008.

PRICE, D. J. S. Network of scientific papers: the pattern of bibliographic reference indicates the nature of scientific research front. Science, New York, v. 149, n. 3683, p. 510-515, Jul. 30, 1965.

ROUSSEAU, R. Journal evaluation: technical and practical issues. Library Trends, Baltimore, v. 50, n. 3, p. 418-439, 2002. 

pesquisa científica no Brasil: estudo exploratório com

\title{
SCIMAGO JOURNAL RANK. Proceedings of the National Academy of
} Sciences of the United States. Disponível em:

$<$ http://www.scimagojr.com/journalsearch.php?q=21121\&tip=sid >. Acesso em: 17 mar. 2015.

SCHWARTZMAN, S. Um espaço para a ciência: a formação da comunidade científica no Brasil. Brasília: Ministério de Ciência e Tecnologia, 2001. Tradução de Sérgio Bath e Oswaldo Biato. Disponível em: <http://www.schwartzman.org.br/simon/spacept/espaco.htm>. Acesso em 23 fev. 2014.

VERGARA, M. R. Ciência e modernidade no Brasil: a constituição de duas vertentes historiográficas da ciência no século XX. Revista da Brasileira de História da Ciência, Rio de Janeiro, v. 2, n. 1, p. 22-31, 2004.

\section{Anais da Academia Brasileira de Ciências and the scientific research in Brazil: an exploratory study based on the $h$-index}

\begin{abstract}
Exploratory study of the journal Anais da Academia Brasileira de Ciências (AABC) of works published duringthe period from 1966 to 2012. The objective is to analyze the variables authorship, gender, affiliation and areas of knowledge that are linked to the authors of the selected articles, identified in the basis Web data of Science (WoS) through the h-index values of the articles AABC in the period. The results indicated that the 33 most cited articles received a total of 1,684 citations, representing $18 \%$ of all citations received by the 4,040 articles identified by WoS in AABC. In this set of articles, single authorship is prevalent, most of the authors are male and are affiliated to Brazilian institutions, especially universities, with the highest incidence of citation related to the area of Biomedical and Medical Sciences. Although the results did not reveal relevant news that change the profile of the current Brazilian scientific production, we sought to promote greater opportunities for reflection and discussion of the results obtained, analyzing peculiarities related to some of the most cited articles and establishing a comparison between general data of the AABC and data from the journal Proceedings of the National Academy of Sciences of the United States.
\end{abstract}


Keywords: Citation analysis. H-index, Authorship. Scientific production. Scientific journals.

${ }^{1}$ Jorge Eduardo Hirsch é físico argentino com PhD pela Univeridade de Chicago e pós-doutorado pelo Kavli Institute de Física Teórica da Universidade da California. Inicialmente, criou o indicador para avaliar a qualidade relativa da produção científica dos físicos teóricos da Universidade da Califórnia em São Diego.

Recebido: 29/07/2014

Aceito: 16/04/2015 